Accessibility to clean water was difficult. $96 \%$ individuals reported not practicing any preventive measure for malaria. Inadequate Chloroquine was available in the medical camps, arthremeter was not available. The doctors reported, visiting of 8-10 patients daily with malaria symptoms.

Conclusion Malaria is a major epidemic and public health concern particularly during flood catastrophe. Transparent policy making is required to design strategies for the preparedness of malaria in Pakistan.

\section{SP3-20 MATERNAL IRON DEFICIENCY ANAEMIA DURING LABOUR AND ITS IMPACT ON BIRTH OUTCOMES IN WESTERN IRAN}

doi:10.1136/jech.2011.1429760.20

N Esmailnasab, ${ }^{*}$ A Afkhamzadeh. Kurdistan University of Medical Sciences, Sanandaj/ Kurdistan, Iran

Introduction Iron deficiency anaemia is one of the most common blood disorders across the world. According to the WHO, more than half of pregnant women and one third of non-pregnant women of childbearing age suffer from anaemia. The present cross-sectional study aimed to determine prevalence of maternal iron deficiency anaemia during labour and its impact on birth outcomes.

Methods A sample of 600 mothers with live births who referred to the Besat hospital in Sanandaj city western Iran was randomly recruited. Blood samples were taken and measured for Maternal $\mathrm{CBC}$ and neonatal $\mathrm{CBC}$ as well as Feretin laboratory tests.

Results Prevalence of iron deficiency anaemia among mothers and their neonates was $22.7 \%$ and $5.8 \%$ respectively with a significant correlation $(p=0.001)$. The mean haemoglobin levels were 11.95 and 11.8 in mothers who received iron supplements during pregnancy and those who did not with no significant association. Moreover, no significant relationship was observed between maternal iron deficiency anaemia and birth outcomes of LBW, prematurity, preterm labour and Apgar score.

Discussion Maternal iron deficiency anaemia was not significantly associated with adverse birth outcomes of LBW, prematurity and low Apgar score in the present study. Laboratory based interventional programs are recommended to improve prenatal care for pregnant women in developing countries' surveillance system.

\section{SP3-21 LANDMINE VICTIMS IN IRAN KURDISTAN; DEMOGRAPHIC FEATURES AND ACCIDENT CHARACTERISTICS}

doi:10.1136/jech.2011.1429760.21

${ }^{1} \mathrm{~A}$ Afkhamzadeh, ${ }^{*} \mathrm{~A}$ Delpisheh, ${ }^{3} \mathrm{H}$ Mohammadzadeh. ${ }^{1}$ Kurdistan University of Medical Sciences, Sanandaj/Kurdistan, Iran; ${ }^{2}$ llam University of Medical Science, Ilam, Iran; ${ }^{3}$ Payam Nour University of Kamyaran, Kamyaran, Iran

Background Iranian civilians living in border areas are still victims of un-neutralised war mines. This cross-sectional study aimed to assess demographic characteristics and features associated with the incident in a representative group of victims.

Materials and Methods Overall, 300 civilian mine victims in Kurdistan Province western Iran during 1991-2005 through a cluster randomised sampling method were recruited. The documentary data for those who lost their lives was gathered from the archives of local police, Red Crescent and War-disabled Organisation. Other survived mine-injured victims were interviewed for data collection using a validated checklist. SPSS was used for all analyses.

Results Overall, $17.7 \%$ of victims $(n=53)$ have been killed immediately after explosion and $82.3 \%(n=247)$ were injured. Of those survived, 40\% ( $n=99)$ had an amputation surgery of lower limb in particular. Majority of victims were either farmers, shepherd mans, or children. Among risk factors investigated, victims' job and age were significantly correlated with mine accidents in Kurdistan province after adjusting for other factors including gender, education level and socioeconomic status (Adjusted OR 2.1, 95\% CI 1.1 to 3.2, $\mathrm{p}<0.01$ and AOR 1.7, 95\% CI 1.1 to $2.5, \mathrm{p}=0.04$ respectively).

Conclusions Young civilians living in border areas between Iran and Iraq with certain jobs are still affected by un-neutralised war mines despite the grate efforts made by the government. Struggling efforts are required to avoid and minimise the adverse effects of mine expulsions in border areas in west of country and in Kurdistan in particular

\section{SP3-22 CHILD INJURY PREVENTION IN VIETNAM: ACHIEVEMENTS AND CHALLENGES}

doi:10.1136/jech.2011.142976o.22

${ }^{1} \mathrm{~S}$ Boufous, ${ }^{*}{ }^{1} \mathrm{M}$ Ali, ${ }^{2} \mathrm{H}$ Nguyen, ${ }^{3} \mathrm{M}$ Stevenson, ${ }^{1} \mathrm{R}$ Ivers, ${ }^{2} \mathrm{C}$ P Viet, ${ }^{4} \mathrm{~A}$ Nguyen. ${ }^{1}$ The George Institute for Global Health, University of Sydney, Sydney, New South Wales, Australia; ${ }^{2}$ Hanoi School of Public Health, Hanoi, Vietnam; ${ }^{3}$ Department of Epidemiology and Preventive Medicine, Monash University, Mebourne, Australia; ${ }^{4}$ The United Nations Children's Fund, Hanoi, Vietnam

Introduction As with other South Asian countries, injury is becoming a leading cause of death and morbidity among children in Vietnam. In response, government and non-government agencies in Vietnam have combined efforts during the last decade to develop and implement various child injury prevention strategies and programs. This study provides an overview of the burden of child injury, reviews relevant prevention strategies, and makes recommendations for child injury prevention in Vietnam.

Methods A review of available information related to child injury prevention in Vietnam, including peer reviewed studies, policy documents and reports from various sources, was conducted using commonly utilised databases (Medline, CINAHL, Cochrane Library, etc). Relevant grey literature was also solicited during semi-structured interviews with representatives of key stakeholders, including Ministry of Health, Ministry of Labor, WHO, UNICEF, Vietnam Red Cross, Counterpart International, Hanoi School of Public Health.

Results Drowning is the leading cause of fatal injury in Vietnamese children followed by road traffic crashes, falls, poisoning, burns and animal bites. There have been notable achievements in terms of increasing awareness of injury facing children at all levels in the community and developing a sound injury prevention policy framework in a relatively short period of time. However, much needs to be done to implement necessary environmental and legislative changes, strengthen child injury surveillance and injury prevention research; and to improve access to health services.

Conclusion The insight into the experience of Vietnam could benefit other low and middle income countries with a high burden of child injury.

\section{SP3-23 RECOGNITION OF THE OCCURRENCE OF STROKE FROM AMBULANCE CALLS}

doi:10.1136/jech.2011.1429760.23

K Ohshige. ${ }^{*}$ Yokohama National University, Yokohama, Kanagawa, Japan

Introduction Intravenous administration of tissue plasminogen activator (tPA) is effective for acute ischaemic stroke. Since there is a critical time of only a few hours to start such therapy, it is important to identify patients with stroke as soon as possible. This study aimed to establish a recognition algorithm for stroke occurrence at the time of emergency calls.

Methods The data used in the study was collected from the Yokohama emergency triage system from 1 January to 30 June 2010. In 